\title{
Physical limits of the ballistic and nonballistic spin-field-effect transistor: Spin dynamics in remote-doped structures
}

\author{
E. Ya. Sherman ${ }^{1}$ and Jairo Sinova ${ }^{2}$ \\ ${ }^{1}$ Department of Physics, University of Toronto, 60 St. George Street, Toronto, Ontario, Canada M5S 1A7 \\ ${ }^{2}$ Department of Physics, Texas A\&M University, College Station, Texas 77843-4242, USA \\ (Received 20 January 2005; revised manuscript received 24 June 2005; published 5 August 2005)
}

\begin{abstract}
We investigate the spin dynamics and relaxation in remotely doped two dimensional electron systems where the dopants lead to random fluctuations of the Rashba spin-orbit coupling. Due to the resulting random-spin precession, the spin-relaxation time is limited by the strength and spatial scale of the random contribution to the spin-orbit coupling. We concentrate on the role of the randomness for two systems where the direction of the spin-orbit field does not depend on the electron momentum, the spin-field-effect transistor with balanced Rashba and Dresselhaus couplings and the (011) quantum well. Both of these systems are considered as promising for the spintronics applications, because the suppression of the Dyakonov-Perel' mechanism there makes the realization of a spin-field-effect transistor in the diffusive regime possible. We demonstrate that the spin relaxation through the randomness of spin-orbit coupling imposes important physical limitations on the operational properties of these devices.
\end{abstract}

DOI: 10.1103/PhysRevB.72.075318

PACS number(s): 73.63.-b, 85.75.-d

\section{INTRODUCTION}

The simultaneous control of the spin and charge dynamics in low-dimensional semiconductors by electrical means has been one of the major goals of the semiconductor spintronics field for over a decade. ${ }^{1,2} \mathrm{~A}$ strong focus within this field has been systems with spin-orbit (SO) coupling, which connects both the spin and charge degrees of freedom. In strongly asymmetric heterostructures grown along the [001] direction, the dominant SO coupling is of the Rashba type, due to the inversion asymmetry of the confining potential, ${ }^{3,4}$ and has the form $H_{R}=\alpha_{R}\left(\sigma_{x} p_{y}-\sigma_{y} p_{x}\right) / \hbar$, acting as a momentumdependent Zeeman field that aligns the spins of electrons perpendicular to their in-plane momentum. Here, $\sigma$ are the Pauli matrices, and $\boldsymbol{p}=\left(p_{x}, p_{y}\right)$ is the in-plane momentum. The controlled precession of injected spin-polarized carriers around this effective Zeeman field produced by the Rashba coupling in momentum space is the basis for the seminal proposal of Datta and Das of a ballistic spin-field-effect transistor (DD-SFET). ${ }^{5}$ However, a decade-long quest to realize such a device has yielded disappointing results. ${ }^{1,2}$ In part, this is due to the fact that the SO coupling, which crucially influences spin transport, also determines the spin-relaxation rate in weakly disordered systems through random precession due to elastic-scattering processes-the so-called Dyakonov-Perel' (DP) mechanism ${ }^{6}$ - hence limiting the validity of the two-dimensional DD-SFET to the ballistic regime.

Schliemann et $a l .{ }^{7}$ have recently suggested a way to design a SFET in (001)-plane-based structures that should work on the diffusive regime, hence without the need to reach this ellusive ballistic regime, by using the interplay between the Rashba and the Dresselhaus SO coupling, $H_{D}=\alpha_{D}\left(\sigma_{y} p_{y}\right.$ $\left.-\sigma_{x} p_{x}\right) / \hbar$, which originates from the lack of inversion symmetry in the unit cell of the host material..$^{8,9}$ The key aspect of this proposed device is that the momentum dependence of the direction of the effective Zeeman field is removed if the
Rashba and Dresselhaus coupling strengths are equal with $\alpha_{R}= \pm \alpha_{D}$. In this case, the in-plane SO field is parallel either to the [110] or [1 $1 \overline{1} 0]$ direction and does not depend on the direction of the electron momentum. It was suggested recently in Refs. 10 and 11 that the freezing of one of the electron-momentum components in one-dimensional channels suppresses the DP mechanism of spin relaxation and leads to a much longer spin lifetime than in the $2 \mathrm{D}$ quantum wells. This idea, is, however, different from the proposal of Ref. 7, where the DP mechanism becomes much less efficient through the combined effects of the Rashba and Dresselhaus terms (see also in Ref. 12).

By decoupling the momentum and the spin-quantization axis orientation, the DP mechanism is greatly reduced, and hence the spin-coherence time increased, making such a device apparently realizable in both the diffusive and ballistic regimes. The condition $\alpha_{D}=\alpha_{R}$ is indeed a possible one, since it has been recently demonstrated that by applying external bias across the quantum well $(\mathrm{QW})$, one can change the magnitude of $\alpha_{R}$ in InGaAs/InAlAs-based ${ }^{13,14}$ and GaAs/AlAs-based ${ }^{15-17}$ structures and reach ratios of $\alpha_{D} / \alpha_{R}$ close to one. Another example of the decoupled SO field and momentum directions is provided by (011) zinc-blende quantum wells, where the Dresselhaus SO coupling leads to the effective field perpendicular to the well plane at arbitrary electron momentum, as discussed later.

Here we consider an aspect of the practical realization of this device which will have important limiting consequences in its operating properties. We show that the randomness of SO coupling arising in doped systems destroys the regular precession of electron spins and leads to a spin relaxation both in the SFET device and the (011) quantum wells even in the ballistic regime. We show that the size of the devices based on the idea of this effective SO decoupling is limited even in the best possible realizations of the devices, and therefore, the spins cannot be coherently transferred at arbitrary distances. In most of the low-dimensional QW-based 
semiconducting systems, the SO coupling and finite concentration of electrons are achieved either by one-side or asymmetric two-side remote doping at distances between the symmetry plane of the QW and the dopant layer of the order of $10 \mathrm{~nm} .{ }^{13,14,16}$ The spatial nonuniform distribution of dopant ions leads to fluctuations of the SO coupling, and therefore, the condition of $\alpha_{D}=\alpha_{R}$ in a SFET can only be achieved on the average throughout the sample; the fluctuations of this coupling are analogous to the classical "shot" noise in the transport properties. The effect is, in some aspects, similar to that investigated by Mel'nikov and Rashba in doped bulk Si and Ge. ${ }^{18}$ At the same time, recent theoretical analysis ${ }^{19}$ shows that in the case of strong SO coupling (large $\alpha_{R}$ ) achieved in highly asymmetric heterosructures, the condition $\alpha_{D}=\alpha_{R}$ is hardly achievable by applying a bias, and therefore, we are left with the quantum wells where the moderate Rashba coupling arises due to the doping or weak structural asymmetry. For a comparison to possible experimental realizations, we shall consider InGaAs structures, where the Rashba coupling can be reliably influenced in a wide range by the applied bias, as has been proven in Refs. 13 and 14 .

We organize the rest of the article as follows: In Sec. II, we discuss the properties of the SO coupling considered. In Sec. III, we discuss the spin relaxation in the proposed fieldeffect transistor (FET) with balanced Rashba and Dresselhaus SO coupling terms, and in Sec. IV, the one for the (011) quantum well. In Sec. V, we present conclusions for these results.

\section{RANDOMNESS OF THE ZEEMAN SO FIELD}

We consider a two-dimensional (2D) channel and a very thin dopant layer ( $\delta$ doping) separated by a distance $z_{0}$ with a two-dimensional concentration $n(\mathbf{r})=\Sigma_{j} \delta\left(\mathbf{r}-\mathbf{r}_{j}\right)$ of dopants at points $\mathbf{r}_{j}$ with charge $e$ and $\mathbf{r}=\left(r_{x}, r_{y}\right)$ being the 2D inplane vector.

Since we are interested in quantum wells, where the Rashba SO coupling arises due to an asymmetric doping, we assume that the effect of the electric field of dopants on the SO strength is linear in the $z$ component of the field $E_{z}(\boldsymbol{\rho})$, that is, $\alpha_{R}(\boldsymbol{\rho})=\alpha_{\mathrm{SO}} e E_{z}(\boldsymbol{\rho})$. Here, $\boldsymbol{\rho}$ is a point at the plane of a sufficiently narrow $2 \mathrm{D}$ channel and $\alpha_{\mathrm{SO}}$ is a phenomenological system-dependent parameter. ${ }^{20}$ This relationship takes into account the linear in $E_{z}$ polarization of the electron wave function as well, as found in Ref. 21. In more asymmetric heterostructures or asymmetrically grown quantum wells, the structural asymmetry will lead to a regular coordinateindependent shift $\alpha_{0}$ to the Rashba term, which has no influence on the effects of the randomness. The $z$ component of the Coulomb field due to the dopant ions is given by

$$
E_{z}(\boldsymbol{\rho})=\frac{e z_{0}}{\epsilon} \sum_{j} \frac{1}{\left[\left(\boldsymbol{\rho}-\mathbf{r}_{j}\right)^{2}+z_{0}^{2}\right]^{3 / 2}},
$$

where $\epsilon$ is the dielectric constant, and the summation is performed over the dopant layer.

Figure 1 presents the pattern of $\alpha_{R}(\boldsymbol{\rho})$ at the spatial scale of $200 \mathrm{~nm}$ for $z_{0}=20 \mathrm{~nm}$ obtained by a Monte Carloproduced "white-noise" distribution of the dopant ions. ${ }^{23}$ The

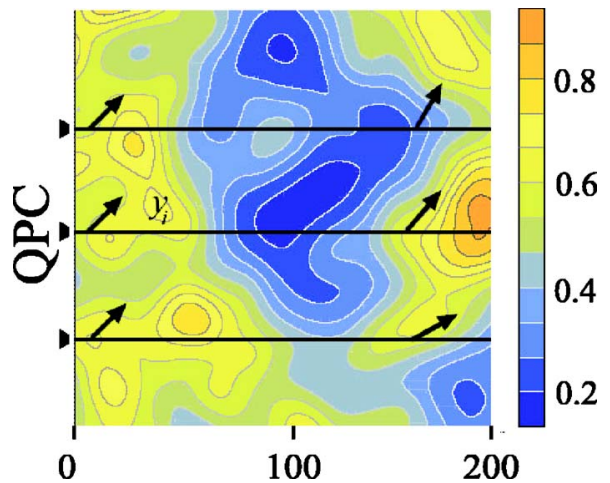

FIG. 1. (Color online) A plot of a typical realization of the random Rashba SO coupling (in arbitrary units) arising due to random variations in the dopant concentration. $z_{0}=20 \mathrm{~nm}$, and the size of the template is $200 \times 200 \mathrm{~nm}$. The quantum point contacts are marked by black triangles, thin lines show the electron paths, and black arrows show the direction of the electron spins.

fluctuations of $\alpha_{R}(\boldsymbol{\rho})$ correlated on the spatial scale of the order of $z_{0}$ become smaller and smoother with the increase of the distance to the dopant layer, as is shown by onedimensional cuts in Fig. 2.

The Rashba parameter is a sum of the mean value produced by the asymmetric field $\langle\alpha\rangle$ and a random contribution with zero mean

$$
\alpha_{R}(\boldsymbol{\rho})=\langle\alpha\rangle+\alpha_{\mathrm{r}}(\boldsymbol{\rho}),
$$

with $\langle\alpha\rangle=\alpha_{0}+2 \pi \alpha_{\mathrm{SO}}\left(e^{2} / \epsilon\right) \bar{n}$, where $\bar{n}$ is the mean value of the 2D dopant concentration. For the $\delta\left(z+z_{0}\right)$ doping in quantum wells with $\alpha_{0}=0$, the relative amplitude of the fluctuations $\sqrt{\left\langle\alpha_{\mathrm{r}}^{2}\right\rangle} /\langle\alpha\rangle=1 / \sqrt{8 \pi \bar{n} z_{0}^{2}} \cdot 22,24$ In contrast to the regular term, which demonstrates a dependence $\alpha_{R}(V)$ on the bias $V$ applied across the well, this random contribution remains relatively weakly influenced and, therefore, cannot be removed.

\section{SPIN RELAXATION IN THE NONBALLISTIC SFET CONDITION}

The randomness in the direction of the spin-precession axis can be removed when at some applied bias $V$, the con-

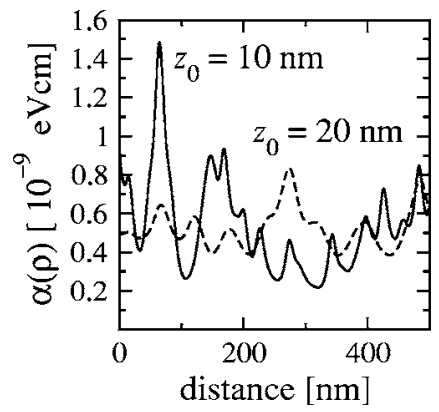

FIG. 2. Typical realization of the random Rashba SO coupling for different distances between the dopant layer and the symmetry plane of the QW as marked near the plots, $\bar{n}=2.5 \times 10^{11} \mathrm{~cm}^{-2}$. 
dition $\alpha_{D}= \pm \alpha_{R}(V)$ is satisfied and $\alpha_{R}(V)$ is spatially uniform. Therefore, the direction of the linear in the in-plane momentum Zeeman field does not depend on the momentum directions, and the spin precession becomes more regular. Here a new integral of motion $\Sigma=\left\langle\sigma_{x}+\sigma_{y}\right\rangle / \sqrt{2}$ appears and supports the regularity of spin dynamics. ${ }^{7}$ We emphasize here that the exact matching is possible only at the limit of zero electron momentum, where both the Rashba and Dresselhaus Hamiltonians are linear in $p$. At finite momenta, the Dresselhaus Hamiltonian must be supplemented by the bulk $k^{3}$-like term, which in the (001) quantum well has the form $\alpha_{c} k_{y} k_{x}\left(\sigma_{x} k_{y}-\sigma_{y} k_{x}\right)$, where $\alpha_{c}$ is the Dresselhaus constant for the bulk, and $\mathbf{k}=\mathbf{p} / \hbar$ is the wave vector. The role of this term depends considerably on the quantum-well width. This effect, as well as the possible but not yet well known momentum dependence of the Rashba parameter, can hamper the ability to build a functioning SFET even if the linear contributions are balanced.

The total Hamiltonian describing the system written in the Hermitian form is

$$
\begin{gathered}
H_{\mathrm{SO}}=H_{D}+H_{R}, \\
H_{R}=\left\langle\alpha_{R}\right\rangle\left(\sigma_{x} k_{y}-\sigma_{y} k_{x}\right)+\frac{1}{2}\left(\sigma_{x}\left\{k_{y},\left[\alpha_{R}(\boldsymbol{\rho})-\left\langle\alpha_{R}\right\rangle\right]\right\}\right. \\
\left.-\sigma_{y}\left\{k_{x},\left[\alpha_{R}(\boldsymbol{\rho})-\left\langle\alpha_{R}\right\rangle\right]\right\}\right), \\
H_{D}=\alpha_{D}\left(\sigma_{y} k_{y}-\sigma_{x} k_{x}\right)+\alpha_{c} k_{y} k_{x}\left(\sigma_{x} k_{y}-\sigma_{y} k_{x}\right),
\end{gathered}
$$

where $\left\{k_{i}, \alpha_{R}(\rho)\right\}$ stands for an anticommutator. The Dresselhaus coupling constant $\alpha_{D}=\alpha_{c}\left\langle k_{z}^{2}\right\rangle$, where $\left\langle k_{z}^{2}\right\rangle$ is the expectation value of the $k_{z}^{2}=-\partial^{2} / \partial z^{2}$ operator in the ground state, and $\alpha_{c} \approx 25 \mathrm{eV} \AA^{3}$ in GaAs- and InAs-based structures. ${ }^{25} \mathrm{In}$ the case of the rigid walls of the QW with the width $w$, $\left\langle k_{z}^{2}\right\rangle=(\pi / w)^{2}$.

Both the cubic terms in the Hamiltonian and the randomness of SO coupling lead to the spin relaxation in the SFET device. Our main interest in this paper is the role of the randomness. However, to find the regime where the effect of the randomness dominates, we will first discuss the contribution of the $\alpha_{c} k_{y} k_{x}\left(\sigma_{x} k_{y}-\sigma_{y} k_{x}\right)$ term for the spin relaxation in the quantum well in the case $\alpha_{D}=\left\langle\alpha_{R}\right\rangle$. We will use the approach developed in Refs. 6,8 , and 12, where spin relaxation is described by

$$
\frac{d \sigma_{i}}{d t}=-\sum_{j} \Gamma_{i j} \sigma_{j},
$$

with $\Gamma_{i j}$ being the anisotropic spin-relaxation tensor. For the relaxation of $\Sigma$ (expected to be conserved in the $\alpha_{D}=\left\langle\alpha_{R}\right\rangle$ case), we obtain

$$
\frac{d \Sigma}{d t}=-\Gamma_{D} \Sigma,
$$

where the contribution of the $k^{3}$-originated terms yields $\Gamma_{D}$ $=\alpha_{c}^{2} k^{6} \tau_{k} / 8 \hbar^{2}$, with $\tau_{k}$ being the momentum-relaxation time. We will demonstrate that, under realistic conditions, this term is much smaller than the contribution of randomness of the SO coupling, which we consider next.

The randomness of the SO coupling discussed here makes the precession irregular and, therefore, pushes the range of parameters in which such a SFET can be realized toward the ballistic regime. We consider a SFET configuration where electrons are injected through perfect nanoscale quantumpoint contacts $^{7}$ (located at points $x=0, y=y_{i}$, where $i$ $=1,2, \ldots, N$ numerates the contacts and the electrons) and then move along the $x$ axis as shown in Fig. 1, interacting with the random SO field. The initial spin state of all electrons is

$$
\phi_{i}(t=0, x=0)=\frac{1}{\sqrt{2}}\left(\begin{array}{c}
1 \\
e^{i \pi / 4}
\end{array}\right),
$$

with all the spins initially polarized along the $x y$ direction. The shape of the injected electron density along the $y$ axis, $\left|\psi\left(y-y_{i}\right)\right|^{2}$, formed by the quantum contact is conserved during the electron propagation such that the corresponding wave function has the form $\Psi_{i}(x, y)=\psi\left(y-y_{i}\right) \exp \left(i k_{x} x\right)$. We assume that the $y$-axis spatial distribution of the wave function is much less than $z_{0}$, and therefore, the spin of the $i$ th electron interacts with the local Rashba field $\alpha_{R}\left(x, y_{i}\right)$ and its spatial derivatives. To estimate the maximum spin-coherence lifetime, we assume that the movement is ballistic, that is, the momentum of injected electron is conserved, and therefore, only the randomness of $\alpha_{R}(x, y)$ leads to the spin decoherence, thus presenting the spin-relaxation mechanism in relatively high-mobility structures with the electron-free path much larger than $10 \mathrm{~nm}$. This restriction implies that the electron kinetic energy is much larger than the potential fluctuations and ignores quantum-mechanical interference effects, which are small in the larger system sizes considered here. Relaxation of this approximation will further decrease the spin-coherence lifetime in the diffusive regime.

Under these conditions, the random contribution to the SO Hamiltonian obtained with Eq. (3) is

$$
H_{\mathrm{r}}=-\alpha_{\mathrm{r}}(\boldsymbol{\rho}) \sigma_{y} k_{x}+\frac{i}{2}\left[\sigma_{y} \frac{\partial \alpha_{\mathrm{r}}(\boldsymbol{\rho})}{\partial x}-\sigma_{x} \frac{\partial \alpha_{\mathrm{r}}(\boldsymbol{\rho})}{\partial y}\right] .
$$

The spin of a moving electron is, therefore, a subject of a randomly time-dependent SO field, with each electron probing the realization of the field at the given path or different electrons probing the different configurations of essentially the same random field. The direction and the magnitude of the Zeeman field randomly change in time, and therefore, a dephasing of the spin states occurs. The correlation function of the random field is characterized by the Fourier component $\left\langle\alpha_{\mathrm{r}}(0) \alpha_{\mathrm{r}}(\tau)\right\rangle_{\omega}$ extended in the range of frequencies $\omega$ up to $\Omega_{d} \sim 1 / \tau_{d}$ where $\tau_{d}=z_{0} / v$ is the time the electron takes to travel a distance $z_{0}$ through which the Rashba coupling changes appreciably, and $v$ is the electron velocity. The frequency $\Omega_{d}$ is much larger than the spin-flip frequency $\Omega_{\mathrm{SO}}$ $\sim \alpha_{R} k / \hbar$, with $\Omega_{\mathrm{SO}} / \Omega_{d}$ being of the order of 0.1 . This condition implies that the random contribution to the Zeeman field is out of resonance with the spin-flip transitions, and for this reason, the spin-flip transition rate is low. Therefore, under these conditions it is necessary to pass through many 


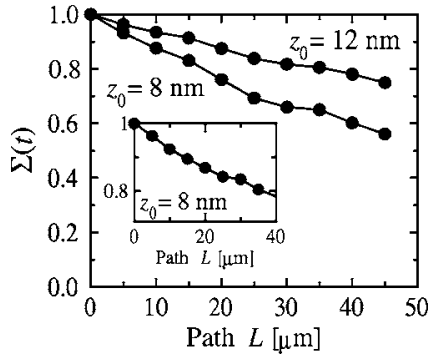

FIG. 3. Dependence of $\left\langle\sigma_{x}+\sigma_{y}\right\rangle / \sqrt{2}$ on the electron path $L$, $\left\langle\alpha_{R}(V=0)\right\rangle=6.0 \times 10^{-10} \mathrm{eV} \mathrm{cm}$. The simulation includes $N=64$ electrons. It is assumed that the regular part of the Rashba term is reduced to 0.6 of its initial value $\left[\left\langle\alpha_{R}(V)\right\rangle=0.6\left\langle\alpha_{R}(V=0)\right\rangle\right]$ by the applied bias. The wave vector of electron $k=0.5 \times 10^{6} \mathrm{~cm}^{-1}$, and the concentration of dopant ions is $\bar{n}=5 \times 10^{11} \mathrm{~cm}^{-1}$. The inset shows spin relaxation at $\bar{n}=2.5 \times 10^{11} \mathrm{~cm}^{-1}, z_{0}=8 \mathrm{~nm}$.

domains of the SO coupling for $\Sigma$ to be destroyed. Analysis similar to that done in Refs. 22 and 26 shows that in the case $\Omega_{d} \gg \Omega_{\mathrm{SO}}$, the random SO-coupling mechanism leads to the spin-relaxation rate of the order of $2 \pi\left\langle\alpha_{\mathrm{r}}^{2}\right\rangle z_{0} \max \left(k^{2}, z_{0}^{-2}\right) / \hbar^{2} v$. In the system considered here, with $k^{2} \gg z_{0}^{-2}$, it scales with the system parameters as $\left\langle\alpha_{R}^{2}(V\right.$ $=0)\rangle / \bar{n} z_{0} k$. This trend is seen in Fig. 3, which presents the results of the Monte Carlo simulation of the spin dynamics. As one can see in Fig. 3, $\left(\left\langle\sigma_{x}\right\rangle+\left\langle\sigma_{y}\right\rangle\right) / \sqrt{2}$ is gradually destroyed at the electron path $L_{s}$ of the order of $50 \mu \mathrm{m}$, that is, about $5 \times 10^{3}$ domains of the SO random coupling. We associate this decay time with the spin-coherence lifetime in the system. The role of the randomness is demonstrated by the fact that the spin-coherence lifetime depends very strongly on the distance between the dopant layer and the conducting electrons increasing with the distance $z_{0}$. Even when the mean SO coupling is the same, the increase in the distance between the dopant layer and the symmetry plane of the quantum well decreases the randomness and, in turn, the spin relaxation rate, as can be seen from a comparison of the $\Sigma(t)$ dependences in Fig. 3. The noise in the $\Sigma(t)$ dependence corresponds to the simulation with a finite number of electrons and must be experimentally observable in the SFETs operating in this mode. For these reasons, the size of the transistor base cannot be very long, and the spins cannot be controllably delivered at distances much larger than $100 \mu \mathrm{m}$ at best.

An order-of-magnitude estimate of the ratio of the relaxation rates due to these two mechanisms, namely, the SO coupling randomness $\Gamma_{\mathrm{r}}$ and the $k^{3}$-term contributions $\Gamma_{D}$, gives

$$
\frac{\Gamma_{\mathrm{r}}}{\Gamma_{D}} \sim 16 \pi \frac{\left\langle\alpha_{\mathrm{r}}^{2}\right\rangle}{\langle\alpha\rangle_{R}^{2}}\left(\frac{\pi}{w k}\right)^{4} \frac{\tau_{d}}{\tau_{k}} .
$$

The small ratio $\tau_{d} / \tau_{k}$, which strongly depends on the mobility, is typically of the order of $10^{-2}$ and, therefore, favors the role of the $k^{3}$ Dresselhaus contribution. However, at $w k<1$, it is compensated by the $\pi^{4}$ prefactor arising due to the size quantization and by the effect that due to the fast in-plane angular dependence of the $k^{3}$-originated terms, the efficiency of this mechanism is decreased, leading to a large prefactor $16 \pi$ in Eq. (8). The exact value of this prefactor is modeldependent, being, however, of the order of 10 at any reasonable model. Therefore, even at relatively small fluctuations of the Rashba SO coupling $\left\langle\alpha_{\mathrm{r}}^{2}\right\rangle /\langle\alpha\rangle_{R}^{2} \sim 0.1$, they will dominate as the spin-relaxation mechanism. We mention here that despite the spin-orbit coupling in our model depending linearly on the momentum, in the quasiballistic regime, the spin-relaxation path $L_{s}=\hbar k / m \Gamma_{\mathrm{r}}$, where $m$ is electron effective mass, shows only a weak electron-momentum dependence. The reason is that the random contribution to the spinprecession angle for passing through one domain, being of the order of $\alpha_{\mathrm{r}} k / \hbar \times z_{0} /(\hbar k / m)$, is momentum independent, and therefore, the number of the domains contributing into $L_{s}$ depends weakly on $k$. At the same time, for the Dresselhaus $k^{3}$ contribution, one would expect $L_{s} \sim k^{-5}$.

\section{THE (011) QUANTUM WELL}

Another interesting system where the direction of the SO field is momentum-independent is the (011) zinc-blende quantum well. Here SO coupling is described by the Dresselhaus Hamiltonian

$$
H_{D}=\alpha_{D} k_{y} \sigma_{z}\left[1-\left(k_{y}^{2}-2 k_{x}^{2}\right) /\left\langle k_{z}^{2}\right\rangle\right],
$$

with the direction of the SO field being always parallel to the $z$ axis, which is perpendicular to the QW plane. Here $\alpha_{D}$ $=\alpha_{c}\left\langle k_{z}^{2}\right\rangle / 2$. The $\left(k_{y}^{2}-2 k_{x}^{2}\right) /\left\langle k_{z}^{2}\right\rangle$ term in square brackets is the bulk-originated Dresselhaus term, which, contrary to the (001) quantum well, due to the symmetry, does not lead to the change in the SO field direction with the changes in the momentum. When the system is the subject of the regular Rashba Hamiltonian $H_{R}=\left\langle\alpha_{R}\right\rangle\left(\sigma_{x} k_{y}-\sigma_{y} k_{x}\right)$ also, the electron spin precesses around the axis determined by the direction of the $H_{D}+H_{R}$ field with the rate $\Omega=2 \sqrt{\alpha_{D}^{2} k_{y}^{2}+\alpha_{R}^{2} k_{\|}^{2}} / \hbar$, where $k_{\|}^{2}=k_{x}^{2}+k_{y}^{2}$. For the component of the spin initially polarized along the $z$ axis, one obtains

$$
\begin{gathered}
\sigma_{z}(t)=\bar{\sigma}_{z}+A_{\sigma} \cos \Omega t, \\
\bar{\sigma}_{z}=\frac{\alpha_{D}^{2} k_{y}^{2}}{\alpha_{D}^{2} k_{y}^{2}+\alpha_{R}^{2} k_{\|}^{2}}, \\
A_{\sigma}=\frac{\alpha_{R}^{2} k_{\|}^{2}}{\alpha_{D}^{2} k_{y}^{2}+\alpha_{R}^{2} k_{\|}^{2}},
\end{gathered}
$$

where $A_{\sigma}$ is the precession amplitude. Randomness in $H_{R}$ causes fluctuations in the magnitude and direction of the Zeeman SO field acting on the electron spin, both now changing randomly in time. Eventually, this leads to the spin relaxation, which can be also understood as the $\sigma_{z}=1$ to $\sigma_{z}$ $=-1$ spin-flip transitions caused by the random field.

We consider the case of the asymmetrically doped quantum well for which the mean value of the Rashba parameter can be influenced by applied bias. A typical evolution of $\left\langle\bar{\sigma}_{z}(t)\right\rangle$ averaged over an ensemble of electrons for the parameters of spin-orbit coupling typical for $\mathrm{In}_{0.5} \mathrm{Ga}_{0.5}$ As quantum wells is presented in Fig. 4. We consider two cases, a 


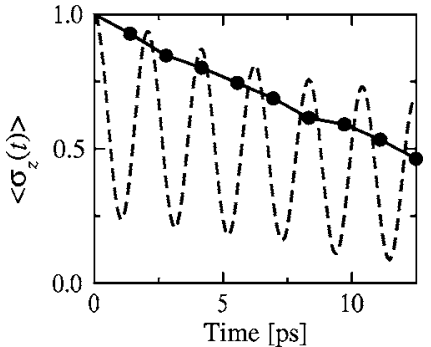

FIG. 4. Time dependence of $\left\langle\sigma_{z}\right\rangle$ for the (011) quantum well. The distance between the QW symmetry plane and dopant layer is $10 \mathrm{~nm}$. The Dresselhaus SO coupling $\alpha_{D}=2.0 \times 10^{-10} \mathrm{eV} \mathrm{cm}$ corresponds to an $\operatorname{In}_{0.5} \mathrm{Ga}_{0.5}$ As with $\alpha_{c}=25 \mathrm{eV} \AA^{3} \mathrm{QW}$, with $w=80 \AA$. The wave vector of electron $k_{y}=2 \times 10^{6} \mathrm{~cm}^{-1}, k_{x}=0, \bar{n}=2.5$ $\times 10^{11} \mathrm{~cm}^{-2}$, and $\left\langle\alpha_{R}(V=0)\right\rangle=3 \times 10^{-10} \mathrm{eV} \mathrm{cm}$. Dashed line represents $\left\langle\alpha_{R}(V)\right\rangle=\left\langle\alpha_{R}(V=0)\right\rangle / 2$; solid line represents $\left\langle\alpha_{R}(V)\right\rangle=0$ (full compensation).

partially compensated and the fully compensated Rashba terms. In the former case, the relaxation occurs as both the amplitude of the oscillations and the mean value averaged over the ensemble of electrons tend to zero. As one can see in the Fig. 4, the decay time of $\left\langle\sigma_{z}(t)\right\rangle$ in the $\operatorname{In}_{0.5} \mathrm{Ga}_{0.5} \mathrm{As}$ structures is of the order of $10 \mathrm{ps,} \mathrm{similar} \mathrm{to} \mathrm{the} \mathrm{case} \mathrm{of} \mathrm{the}$ $\alpha_{D}=\left\langle\alpha_{R}(V)\right\rangle$ transistor.

In "pure" GaAs structures, where $\alpha_{\mathrm{SO}}$ is an order of magnitude smaller than in the $\operatorname{In}_{0.5} \mathrm{Ga}_{0.5} \mathrm{As}$ alloys, one would expect the spin-relaxation time, due to the randomness of the doping, to roughly scale as $\alpha_{\mathrm{SO}}^{-2}$, two orders of magnitude longer, that is, of the order of $10^{3} \mathrm{ps}$. A long electron spincoherence time $T_{s}$ of this order of magnitude has indeed been observed experimentally in Ref. 27 for the (011) symmetrically doped GaAs QWs of relatively low mobility with $\mu$ $\sim 10^{3} \mathrm{~cm}^{2} /(\mathrm{V} \mathrm{s})$. It is instructive to estimate the corresponding distance $L_{s}$ at which the spins of diffusively propagating electrons can be delivered for this time in these low-mobility samples. With this mobility, the momentum-relaxation time $\tau \sim 0.1 \mathrm{ps}$ leads to $L_{s} \sim v\left(T_{s} \tau\right)^{1 / 2}$, where $v \sim 10^{7} \mathrm{~cm} / \mathrm{s}$ is the speed of optically injected electrons investigated in Ref. 27. Here $\left(T_{s} \tau\right)^{1 / 2} \sim 10 \mathrm{ps}$, and therefore, $L_{s}$ is of the order of a few $\mu \mathrm{m}$. Therefore, due to the low mobility, these QWs cannot be used for the simultaneous long-distance charge and spin transfer necessary for the spintronics applications despite a long spin-coherence time there.

\section{CONCLUSIONS}

We have investigated the spin-relaxation rate arising due to a random SO coupling in a possible realization of a SFET with the balanced Rashba and Dresselhaus spin-orbit coupling and the (011) zinc-blende quantum well. These systems, where the direction of the effective spin-orbit Zeeman field is expected to be independent on the electron momentum and, therefore, the Dyakonov-Perel' mechanism of spin relaxation must be suppressed, are considered as promising elements for the spintronics applications. At the same time, due to the randomness of the Rashba contribution to the spin-orbit coupling, causing an additional spin relaxation, in both cases the relaxation time cannot exceed considerably $10 \mathrm{ps}$, thus limiting the size of the base of this transistor of the order of $10 \mu \mathrm{m}$, which weakly depends on the electron momentum, and, therefore, restricts the possibility of their experimental realization. Recent focus in spintronics is related to $\mathrm{Si} / \mathrm{Ge}$ two-dimensional structures, where the spinorbit coupling is weak. ${ }^{22,28-30}$ Within these systems, the randomness of the SO coupling, which is related either to the randomness in the dopant distribution as considered here or to random bonds on the $\mathrm{Si} / \mathrm{Ge}$ interfaces ${ }^{29}$ will cause spin relaxation and limit their potential applications as well.

\section{ACKNOWLEDGMENTS}

E.Ya.S. is grateful to the DARPA SpinS program for financial support and to J. E. Sipe for valuable discussions.
${ }^{1}$ I. Zutic, J. Fabian, and S. Das Sarma, Rev. Mod. Phys. 76, 323 (2004).

${ }^{2}$ Semiconductor Spintronics and Quantum Computation, edited by D. Awschalom, N. Samarth, and D. Loss (Springer, Berlin, 2002).

${ }^{3}$ Yu. A. Bychkov and E. I. Rashba, JETP Lett. 39, 79 (1984); E. I. Rashba, Sov. Phys. Solid State 2, 1874 (1964).

${ }^{4}$ E. I. Rashba, Fiz. Tverd. Tela (Leningrad) 2, 1224 (1960) [Sov. Phys. Solid State 2, 1109 (1960)].

${ }^{5}$ S. Datta and B. Das, Appl. Phys. Lett. 56, 665 (1990).

${ }^{6}$ M. I. Dyakonov and V. I. Perel', Sov. Phys. Solid State 13, 3023 (1972).

${ }^{7}$ J. Schliemann, J. C. Egues, and D. Loss, Phys. Rev. Lett. 90, 146801 (2003).

${ }^{8}$ M. I. Dyakonov and Y. Yu. Kachorovskii, Sov. Phys. Semicond. 20, 110 (1986).

${ }^{9}$ E. I. Rashba and E. Ya. Sherman, Phys. Lett. A 129, 175 (1988); O. Mauritz and U. Ekenberg, Phys. Rev. B 60, R8505 (1999); R.
Winkler, S. J. Papadakis, E. P. De Poortere, and M. Shayegan, Phys. Rev. Lett. 85, 4574 (2000).

${ }^{10}$ A. G. Mal'shukov and K. A. Chao, Phys. Rev. B 61, R2413 (2000).

${ }^{11}$ A. A. Kiselev and K. W. Kim, Phys. Rev. B 61, 13115 (2000).

${ }^{12}$ N. S. Averkiev and L. E. Golub, Phys. Rev. B 60, 15582 (1999).

${ }^{13}$ J. Nitta, T. Akazaki, H. Takayanagi, and T. Enoki, Phys. Rev. Lett. 78, 1335 (1997).

${ }^{14}$ T. Koga, J. Nitta, T. Akazaki, and H. Takayanagi, Phys. Rev. Lett. 89, 046801 (2002).

${ }^{15}$ W. Knap, C. Skierbiszewski, A. Zduniak, E. Litwin-Staszewska, D. Bertho, F. Kobbi, J. L. Robert, G. E. Pikus, F. G. Pikus, S. V. Iordanskii, V. Mosser, K. Zekentes, and Yu. B. Lyanda-Geller, Phys. Rev. B 53, 3912 (1996).

${ }^{16}$ J. B. Miller, D. M. Zumbühl, C. M. Marcus, Y. B. Lyanda-Geller, D. Goldhaber-Gordon, K. Campman, and A. C. Gossard, Phys. Rev. Lett. 90, 076807 (2003).

${ }^{17}$ O. Z. Karimov, G. H. John, R. T. Harley, W. H. Lau, M. E. Flatté, 
M. Henini, and R. Airey, Phys. Rev. Lett. 91, 246601 (2003).

${ }^{18}$ V. I. Mel'nikov and E. I. Rashba, Sov. Phys. JETP 34, 1353 (1972).

${ }^{19}$ E. Shafir, M. Shen, and S. Saikin, cond-mat/0407416 (unpublished)

${ }^{20}$ A detailed kp-theory analysis of the effect of the electric field can be found in E. A. de Andradae Silva, G. C. La Rocca, and F. Bassani, Phys. Rev. B 55, 16293 (1997).

${ }^{21}$ D. Grundler, Phys. Rev. Lett. 84, 6074 (2000).

${ }^{22}$ E. Ya. Sherman, Appl. Phys. Lett. 82, 209 (2003); E. Ya. Sherman, Phys. Rev. B 67, 161303(R) (2003).

${ }^{23}$ For a review of applications of Monte Carlo simulations in theoretical spintronics, see S. Saikin, Yu. V. Pershin, V. Privman, cond-mat/0504001 (unpublished).

${ }^{24}$ A. L. Efros and B. I. Shklovskii, Electronic Properties of Doped Semiconductors (Springer, Heidelberg, 1989).
${ }^{25} \mathrm{R}$. Winkler, in Spin-orbit Coupling Effects in Two-Dimensional Electron and Hole Systems, Springer Tracts in Modern Physics (Springer, Berlin, 2003).

${ }^{26}$ M. M. Glazov and E. Ya. Sherman (unpublished).

${ }^{27}$ S. Döhrmann, D. Hägele, J. Rudolph, M. Bichler, D. Schuh, and M. Oestreich, Phys. Rev. Lett. 93, 147405 (2004); Y. Ohno, R. Terauchi, T. Adachi, F. Matsukura, and H. Ohno, Phys. Rev. Lett. 83, 4196 (1999).

${ }^{28}$ Z. Wilamowski, W. Jantsch, H. Malissa, and U. Rößler, Phys. Rev. B 66, 195315 (2002).

${ }^{29}$ L. E. Golub and E. L. Ivchenko, Phys. Rev. B 69, 115333 (2004).

${ }^{30}$ C. Tahan, and R. Joynt, Phys. Rev. B 71, 075315 (2005); J. L. Truitt, K. A. Slinker, K. L. M. Lewis, D. E. Savage, C. Tahan, L. J. Klein, R. Joynt, M. G. Lagally, D. W. van der Weide, S. N. Coppersmith, M. A. Eriksson, A. M. Tyryshkin, J. O. Chu, and P. M. Mooney, cond-mat/0411735 (unpublished). 